\title{
The Program in Interdisciplinary Studies of the Institute for Advanced Study, Princeton
}

Michael Th. Rassias (University of Zürich, Switzerland) and Olaf Witkowski (Tokyo Institute of Technology, Japan)

The Institute for Advanced Study (IAS), Princeton, is one of the very few places in the world where everyone is totally absorbed in pure research. At the IAS there is no student body, just chalk, blackboards, books and scholars offered complete freedom to think and explore. It was founded in 1930 by Abraham Flexner, Louis Bamberger and Caroline Bamberger Fuld, and since then it has served as the academic home of emblematic figures of science like Albert Einstein, Kurt Gödel, John von Neumann and J. Robert Oppenheimer, to name just a few.

This small academic utopia is also the home of the so-called Program in Interdisciplinary Studies (PIDS), which provides a meeting place for members from the IAS, Princeton, and beyond. These scholars engage in conversations and research projects that address the wider questions that rarely get asked within individual disciplines; questions like, what is the nature of knowledge in different academic areas, in the humanities, the social sciences, the natural sciences and mathematics? How may these fields stimulate each other? And how do such types of knowledge compare with those found in the arts, in the business world, in ancient traditions and in daily life?

The Program started in 2002, as the response to the perceived need to have more communication between 


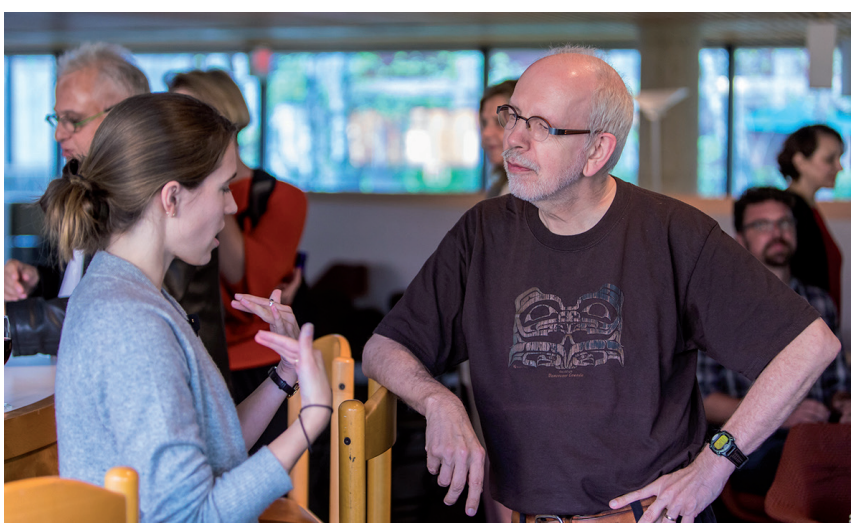

Susan Clark (left) and the Head of the PIDS, Professor Piet Hut (right).

the four main Schools at IAS (Mathematics, History, Natural Science, Social Science), and possibly even collaborations between the Schools. Through PIDS, one can be exposed to various different scientific disciplines and, by engaging in discussions with scientists hailing from a broad spectrum of scientific areas, one can possibly discover unexpected applications and interconnections of one's own field to something completely different. For example, number theorists may interact with nuclear physicists, philosophers with astronomers, musicologists with computer scientists etc., in a very intellectually stimulating environment. The nature of PIDS - attracting members from all Schools within the IAS and beyond - can allure even the purest of researchers, like pure mathematicians whose work may seem distant from other fields, to engage in conversations which often lead to intriguing new interdisciplinary questions. Commencing from concrete problems, discussions often stretch to explorations of a more philosophical flavour, such as for example the emergence of life, the origin of mathematics, or the nature of knowledge.

The output of such collaborative projects from PIDS members ranges from the very pure, like works on Goldbach's conjecture (cf. $[5,11])$ and Riemann's hypothesis (cf. $[6,7])$; or abstract, such as the philosophy of "Math Matter and Mind" (cf. [3]); to more applied, like works on astrophysics (cf. [4]), artificial life simulations (cf. [1,2,12, 13]), and biology (cf. [8]). In a recent volume dedicated to the Riemann zeta function, co-edited by current PIDS visitor Michael Th. Rassias in collaboration with Hugh L. Montgomery and Ashkan Nikeghbali [9], F. Dyson of the IAS writes about a fascinating interdisciplinary approach to Riemann's hypothesis. He argues that one could combine physics and pure mathematics by studying one dimensional quasi-crystals and associating them with the zeros of the Riemann zeta function. In a more playful yet challenging spirit, prompted by conversations with Monica Manolescu and Siobhan Roberts as a PIDS visitor in 2012, Philip Ording published a book, [9] challenging the conception of mathematics as having a single style strictly characterized by symbolic notation and abstraction.

Apart from the individual as well as collaborative research that members of PIDS conduct, there are several outreach and dissemination activities initiated by

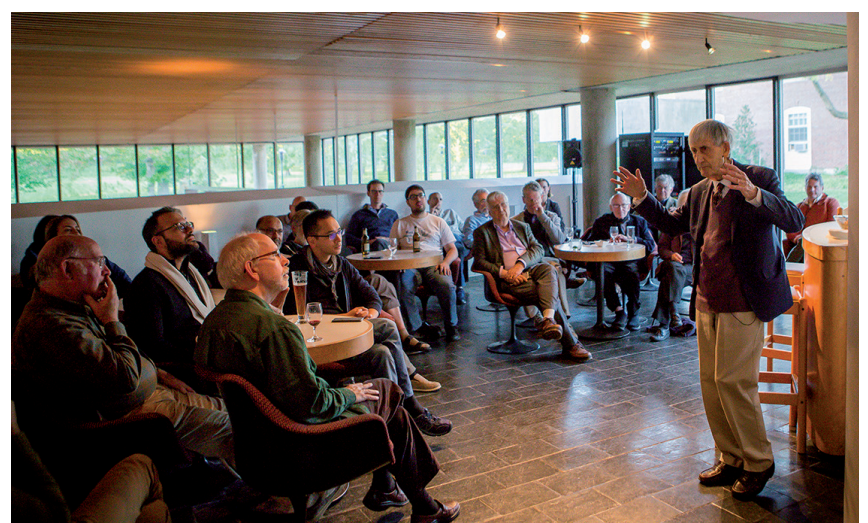

Professor Freeman Dyson's talk in the "After Hours Conversations" of the PIDS, on October 18, 2018.

this Program, taking place at the IAS, Princeton, but also elsewhere, such as in New York, Tokyo, etc. One central facet of these activities are the so-called After Hours Conversations, which constitute informal short talks held in the bar area of the IAS twice a week during the first two months of each semester. These talks are open to faculty, members, visitors, staff, spouses and partners in an effort to encourage cross-discipline communication at the IAS. Professors Piet Hut (head of PIDS), Didier Fassin, Helmut Hofer and Myles Jackson moderate these sessions. In these talks, someone gives an informal presentation of no more than 10 minutes, intended for a general audience. The topic of the talk consists of a brief description of a major open problem in the speaker's field of expertise, together with suggestions for possible future progress with respect to that problem. The talk is then followed by 20 minutes of discussion. Afterwards, the remaining participants are free to mingle in more general discussions in smaller groups, preferably with others not from their own School. The lively and intriguing nature of the After Hours Conversations has been very successful, with prominent figures of science having delivered such brief talks, like famed physicist Freeman Dyson. Inspired by these meetings, the School of Mathematics recently introduced the so-called Mathematical Conversations.

Following in this interdisciplinary spirit, the head of PIDS was also invited to contribute to the experience of PIDS and be one of the founding members for a new research centre within the Tokyo Institute of Technology, working on elucidating the rise of initial life on Earth and its subsequent evolution to complex life. This centre, called the Earth-Life Science Institute (ELSI for short), in order to address both the origin and evolution of life, required putting together and co-ordinating a strongly interdisciplinary hub of researchers from very different backgrounds. Following the experience of PIDS, from astrophysicists, planetary scientists, to geochemists, paleontologists, astrobiologists and computer scientists, all researchers had to learn to form a common language to communicate across fields, and design bridging structures to collaborate efficiently as a diverse research institution.

In another instance of such an initiative spreading further, the head as well as members of PIDS in col- 


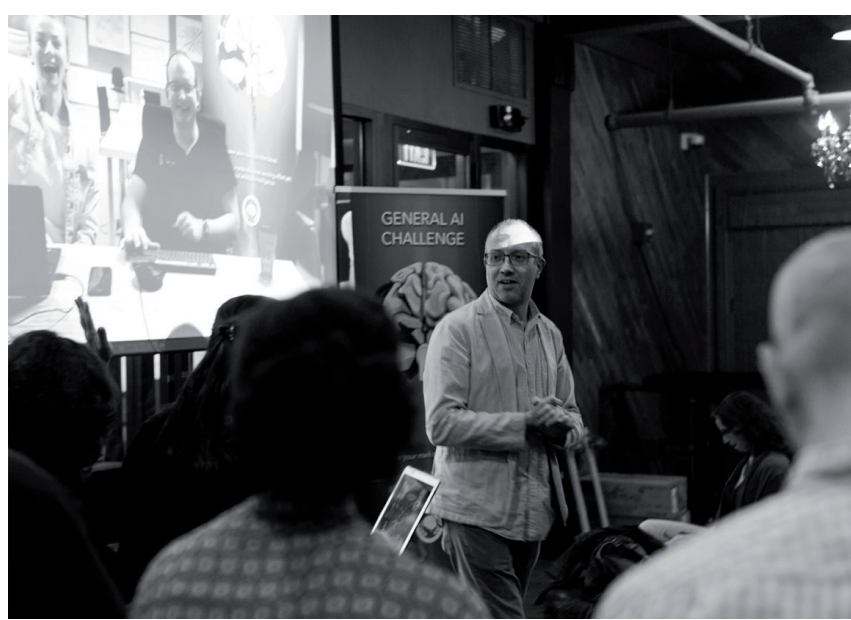

Co-founder of YHouse Professor Caleb Scharf, at YHouse's event "Learning About the Brain and Brain-Inspired Learning", on May 3, 2017.

laboration with scientists from other institutions have also founded a pilot experiment in the form of a virtual research institute called YHouse, which was very active for a couple of years starting in 2016. This design constitutes a unique gathering space ("House") based in Manhattan, New York, dedicated to accelerating our understanding of "why?" questions ("Y") on the nature of awareness through multiple areas of science, technology, and humanities. YHouse's aspiration is to create a culture of open-minded rigour and a spirit of transdisciplinary inquiry that breaks free of the institutional barriers in academia and society.

The presence of a centre as PIDS is essential to the life of an institute, as it not only catalyses work across fields, it also challenges the researcher's area of comfort and fosters very creative work through interdisciplinary exchanges (academic, but also outreach and citizen science). In a sense, it provides a comfortable place to work out of one's comfort zone. This way, PIDS represents an avant-garde initiative in a top institution, which deserves to be considered seriously as a model for future universities and research centres.

\section{Reference}

[1] S.J. Bartlett, O. Witkowski and D. Giovannelli (2017). Cognition and Learning: A Primary Determinant and Seed of Life. In Proceedings of XVIIIth Intl Conf on Origin of Life 2017 (LPI Contrib. No. 1967) at UC San Diego, CA, USA.

[2] Guttenberg, Nicholas, et al. "Permutation-equivariant neural networks applied to dynamics prediction." arXiv preprint arXiv:1612.04530 (2016).

[3] P. Hut, M. Alford, M. Tegmark, On math, matter and mind, Foundations of Physics, 36(6) (2006), 765-794.

[4] P. Hut, S. McMillan, J. Makino, S. Portegies Zwart, Starlab: A Software Environment for Collisional Stellar Dynamics. Astrophysics Source Code Library (2010).

[5] H. Maier and M. Th. Rassias, The ternary Goldbach problem with a prime and two isolated primes, Proceedings of the Steklov Institute of Mathematics, 296(2017), 183-197.

[6] H. Maier and M. Th. Rassias, Explicit estimates of sums related to the Nyman-Beurling criterion for the Riemann Hypothesis, Journal of Functional Analysis, https://doi.org/10.1016/j.jfa.2018.06.022
[7] H. Maier and M. Th. Rassias, On the size of an expression in the Nyman-Beurling-Baez-Duarte criterion for the Riemann Hypothesis, Canadian Mathematical Bulletin, 61(3)(2018), 622-627.

[8] N. Merino, HS. Aronson, D. Bojanova, J. Feyhl-Buska, ML. Wong, S. Zhang, D. Giovannelli, Living at the Extremes: Extremophiles and the Limits of Life in a Planetary Context, EarthArxiv, 2019, doi: 10.31223/osf.io/8eay6

[9] H.L. Montgomery, A. Nikeghbali and M.Th. Rassias (eds.), Exploring the Riemann Zeta Function: 190 years from Riemann's birth, (Preface by Freeman J. Dyson), Springer, 2017.

[10] P. Ording, 99 Variations on a Proof, Princeton University Press, 2019.

[11] M.Th. Rassias, Goldbach's Problem: Selected Topics, Springer, 2017.

[12] O. Witkowski, and T. Ikegami, How to make swarms open-ended? Evolving collective intelligence through a constricted exploration of adjacent possibilities, Special Issue on Open-Ended Evolution, Artificial Life Journal. Volume 25(2), to appear.

[13] O. Witkowski, and T. Ikegami T. Emergence of Swarming Behavior: Foraging Agents Evolve Collective Motion Based on Signaling. PLoS One 11.4: e0152756, 2016.

Michael Th. Rassias is on the Editorial Board of the EMS Newsletter. His photo and CV can be found in previous Newsletter issues.

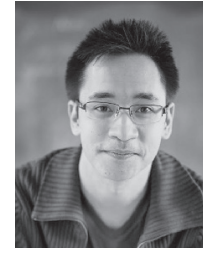

Olaf Witkowski is a Visiting Member at the Institute for Advanced Study, Princeton, and a Research Scientist at the Earth-Life Science Institute. He is also a Chief Scientist at the Cross Research Institute as well as Founding Member of YHouse. His research is in machine intelligence and artificial life. 